\title{
Psoríase e Doença Cardiovascular
}

\author{
Psoriasis and Cardiovascular Disease
}

\author{
Tiago TORRES ${ }^{1,2}$, Rita SALES ${ }^{2}$, Carlos VASCONCELOS ${ }^{2,3}$, Manuela SELORES $^{1,3}$ \\ Acta Med Port 2013 Sep-Oct;26(5):601-607
}

\section{RESUMO}

A psoríase é uma doença inflamatória sistémica crónica, frequente, associada a várias comorbilidades, destacando-se a obesidade, a hipertensão arterial, a diabetes, a dislipidemia e síndrome metabólico. Adicionalmente associa-se também a aumento do risco de doença cardiovascular - enfarte agudo do miocárdio e acidente vascular cerebral. A inflamação sistémica crónica presente na psoríase tem sido sugerida como um factor de risco independente para estas comorbilidades e para o aparecimento de aterosclerose precoce. Esta revisão das várias comorbilidades cardio-metabólicas e do risco de doença cardiovascular associado à psoríase tem como objectivo promover o conhecimento e alertar os clínicos para a necessidade de rastreio, monitorização e tratamento dos factores de risco de doença cardiovascular nestes doentes.

Palavras-chave: Psoríase; Doenças Cardiovasculares; Aterosclerose, Inflamação; Comorbilidade.

\section{ABSTRACT}

Psoriasis is a common, chronic and systemic inflammatory disease associated with several comorbidities, such as obesity, hypertension, diabetes, dyslipidaemia and metabolic syndrome, but also with an increased risk of cardiovascular disease, like myocardial infarction or stroke. The chronic inflammatory nature of psoriasis has been suggested to be a contributing and potentially independent risk factor for the development of cardiovascular comorbidities and precocious atherosclerosis. Aiming at alerting clinicians to the need of screening and monitoring cardiovascular diseases and its risk factors in psoriatic patients, this review will focus on the range of cardiometabolic comorbidities and increased risk of cardiovascular disease associated with psoriasis.

Keywords: Psoriasis; Cardiovascular Diseases; Atherosclerosis; Inflammation; Comorbidity.

\section{INTRODUÇÃO}

A psoríase é uma doença inflamatória crónica, imuno-mediada que afecta aproximadamente 2 a $3 \%$ da população mundial, associada a marcada redução da qualidade de vida dos doentes. ${ }^{1}$

Durante décadas a psoríase foi considerada uma doença primariamente do queratinócito. No entanto, desde a demonstração do efeito terapêutico da ciclosporina em 1979, que passou a ser reconhecida como uma doença imuno-mediada em que tanto a imunidade inata como a adquirida têm um papel preponderante na iniciação e manutenção das lesões cutâneas. ${ }^{2}$ Nos últimos anos tem-se ainda demonstrado que a inflamação na psoríase não é exclusivamente cutânea, mas que existe uma inflamação sistémica, especialmente nos doentes mais graves, com elevação sérica de várias citoquinas inflamatórias, como o TNF- $\alpha, \mathrm{IL}-6$, IL-12, IL-17, IL-20, IL-22 e IL-23. ${ }^{3}$

Vários trabalhos demonstraram maior incidência de obesidade, diabetes mellitus (DM), hipertensão arterial (HTA), síndrome metabólico e doença cardiovascular, como enfarte agudo do miocárdio (EAM) e acidente vascular cerebral (AVC) nos doentes com psoríase..$^{4-7}$ Acresce a esta maior incidência de factores de risco de doença cardiovascular, a inflamação sistémica que parece ter um papel importante no desenvolvimento de aterosclerose e doença cardiovascular $^{8} \mathrm{em}$ particular nas formas mais graves e prolongadas da doença.

Assim, o componente cutâneo da psoríase pode ser, em muitos doentes, apenas a 'ponta do iceberg'. (Fig. 1)

Nesta revisão da literatura, os autores vão abordar as forma clínicas de psoríase e a sua associação com factores de risco cardiovasculares, inflamação sistémica e a hipótese de a psoríase constituir per se um factor de risco de doença cardiovascular.

\section{Formas clínicas de psoríase}

A psoríase caracteriza-se pela presença de placas eritematosas, descamativas, muitas vezes pruriginosas ou dolorosas. Tem um curso crónico com fases de agravamento e de acalmia, raramente com remissões espontâneas. ${ }^{1}$

Existem várias formas: a psoríase em placas é a mais comum e afecta cerca de 80 a $90 \%$ dos doentes. Caracteriza-se por placas eritematosas, bem definidas, de vários tamanhos, habitualmente recobertas por descamação prateada, localizando-se geralmente no couro cabeludo, tron$\mathrm{co}$, cotovelos e joelhos, distribuindo-se frequentemente de forma simétrica. Aproximadamente $80 \%$ dos doentes tem uma forma ligeira, enquanto $20 \%$ tem formas moderadas a graves, com envolvimento de mais de $10 \%$ da superfície corporal, necessitando habitualmente de terapêutica sistémica ou fototerapia. ${ }^{1}$

1. Serviço de Dermatologia. Centro Hospitalar do Porto. Porto. Portugal.

2. Instituto de Ciências Biomédicas Abel Salazar. Universidade do Porto. Porto. Portugal.

3. Unidade de Imunologia Clínica. Centro Hospitalar do Porto. Porto. Portugal.

Recebido: 13 de Maio de 2013 - Aceite: 10 de Setembro de 2013 | Copyright @ Ordem dos Médicos 2013 


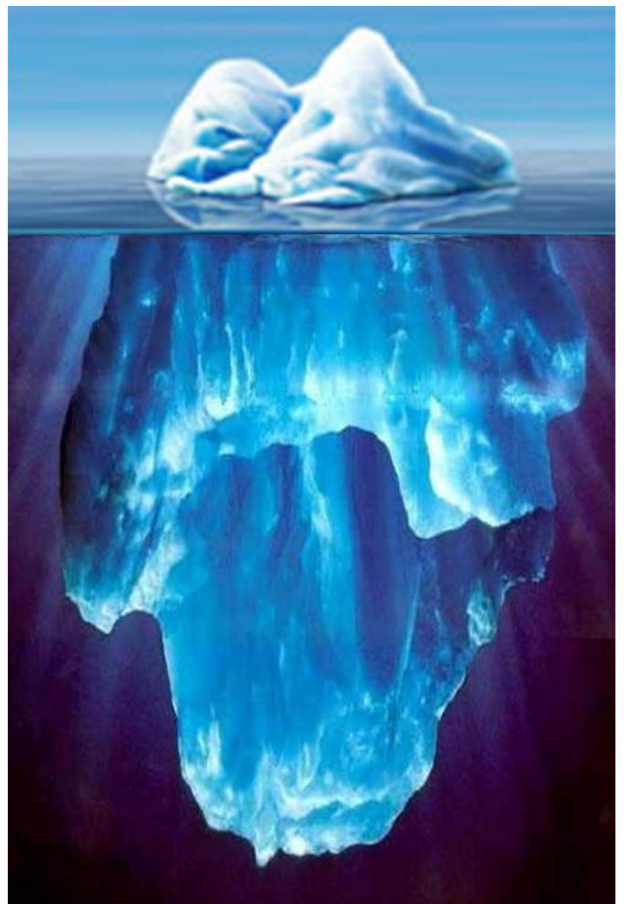

Figura 1 - Comorbilidades associada à psoríase

Na psoríase inversa as lesões localizam-se às pregas cutâneas axilares, inguinais, perineais, interglúteas e infra-mamárias e, devido a maior humidade destes locais, as lesões tendem a ser mais eritematosas e menos descamativas.

A forma gutata caracteriza-se por pequenas pápulas com descamação fina e ocorrem mais frequentemente em indivíduos jovens, sendo muitas vezes desencadeadas por infecções respiratórias superiores por estreptococos $\beta$-hemolíticos do grupo $A$.

Formas mais graves incluem a psoríase pustulosa e psoríase eritrodérmica, em alguns casos necessitando de internamento hospitalar. A psoríase pustulosa caracteriza-se por múltiplas pústulas que podem ser localizadas ou generalizadas. A forma generalizada (variante de von Zumbush) é uma forma aguda, grave, associada a sintomas sistémicos e febre. A psoríase eritrodérmica, manifesta-se por eritema envolvendo mais de $90 \%$ da superfície corporal, habitualmente com sintomas sistémicos.

Em todas as formas pode existir envolvimento ungueal. Este ocorre em mais de $50 \%$ dos doentes, incluindo picotado ungueal, onicólise, hiperqueratose subungueal e mancha de óleo. ${ }^{1}$

\section{Doença Cardiovascular}

A associação entre doença cardiovascular e psoríase foi descrita há quase 50 anos, mas foi na última década que se observou um aumento do interesse e da investigação nesta área. Assim, têm sido publicados vários estudos de coorte e populacionais que têm demonstrado esta associação. ${ }^{4-6,9-11} \mathrm{E}$ se no início, a maioria dos estudos avaliavam apenas a prevalência de factores de risco cardiovasculares, os trabalhos mais recentes estudaram especialmente
Pele

Comorbilidades cardiovasculares

- hipertensão arterial

- diabetes melitus

- dislipidemia

- obesidade

- síndrome metabólico

Aterosclerose precoce

- calcificação coronária

- disfunção endotelial

- espessamento intima-média carotídea

Doença cardiovascular

- enfarte agudo miocárdio

- acidente vascular cerebra

- doença arterial periférica a incidência de eventos cardiovasculares nos doentes com psoríase.

Gelfand et al $^{6}$ reportou, num estudo prospectivo envolvendo 130976 doentes com psoríase e 556995 controlos, que a psoríase se associava a um risco significativamente aumentado de EAM, mesmo efectuando análise multivariada com os vários factores de risco cardiovasculares como diabetes, história familiar de EAM, hipertensão, tabaco e índice de massa corporal. O risco relativo de EAM era maior para doentes jovens com psoríase (<30 anos) com um hazard ratio $(\mathrm{HR})$ de 1,29 e 3,10 para psoríase ligeira e grave respectivamente, enquanto nos mais velhos (>60 anos) o HR era de 1,08 e 1,36.

Noutro estudo com 3236 doentes com psoriáticos e 2 500 controlos, e após ajuste aos vários factores de risco tradicionais de doença cardiovascular, observou-se, não só uma prevalência superior de doença cardíaca isquémica, mas também de doença cerebrovascular e de doença vascular periférica. ${ }^{12}$ Ahlehoff et al ${ }^{13}$ reportou, num estudo efectuado na Dinamarca envolvendo 36765 doentes com psoríase ligeira e 2793 doentes com psoríase grave, um risco relativo de AVC em doentes com menos de 50 anos de $1,97(95 \% \mathrm{Cl}, 1,66-2,34)$ e $2,80(1,81-4,34)$ respectivamente, diminuindo ligeiramente nos doentes com mais de 50 anos [RR 1,13 (95\% Cl, 1,04-1,21) e 1,34 (95\% Cl, 1,041,71)].

Recentemente foi demonstrado aumento da frequência de disfunção eréctil, patologia marcadamente relacionada com aterosclerose, nos doentes com psoríase, apesar de não ter sido mostrado que a psoríase seria um factor de risco independente para disfunção eréctil. ${ }^{14}$

Este aumento de doença cardiovascular em doentes com psoríase poderá resultar de múltiplos mecanismos. Por 
um lado, a elevada incidência de múltiplos factores de risco de doença cardiovascular, como obesidade, hipertensão, dislipidemia e diabetes (provavelmente geneticamente relacionada) e, por outro, a inflamação sistémica existente na psoríase que promove uma aterosclerose precoce. (Fig. 2)

\section{Inflamação e Aterosclerose}

Está bem demonstrado que a inflamação sistémica se associa ao desenvolvimento de aterosclerose. ${ }^{8}$ Assim, considera-se que os doentes com psoríase, especialmente os mais graves, possam ter um risco superior de doença cardiovascular.

De facto, a inflamação sistémica presente na psoríase parece promover uma aterosclerose acelerada de forma independente, através de disfunção endotelial e stress oxidativo, em muito semelhante à observada noutras doenças inflamatórias crónicas sistémicas como o lúpus eritematoso

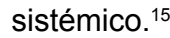

Na psoríase, a inflamação caracteriza-se por elevados níveis de TNF- $\alpha$, INF- $\gamma$, INF- $\alpha$, IL-1, IL-6 e IL-17, produzidos pelas células inflamatórias que infiltram a pele (linfócitos, neutrófilos e células dendrítica) e pelos queratinócitos ativados. ${ }^{3}$ Estas citoquinas têm a capacidade de induzir várias funções pró-aterogénicas do fígado e tecido adiposo, incluído a produção de proteína $C$ reactiva e outras adipoquinas pró-inflamatórias conduzindo a insulino-resistência e disfunção endotelial. ${ }^{16}$

A inflamação aterosclerótica assemelha-se à observada na psoríase, incluindo o perfil de citoquinas e os tipos de células imunológicas intervenientes (ambas as patologias são principalmente mediadas por linfócitos Th1). ${ }^{8} \mathrm{Na}$ psoríase, tal como na aterosclerose ocorre uma desregulação entre Th17 / Treg (linfócitos T reguladores), com aumento da expressão de citoquinas dependentes dos Th17 por diminuição dos Treg. ${ }^{17}$

O impacto clínico tem vindo a ser demonstrado em vários estudos, que demonstraram um aumento da prevalência de aterosclerose em doentes com psoríase. Ludwig et al ${ }^{18}$ mostrou maior prevalência de calcificação arterial coronária em doentes com psoríase, demonstrando igualmente que a psoríase seria um factor de risco independente para calcificação arterial coronária. Muitos outros indicadores de aterosclerose, como a espessura da íntima-média da artéria carotídea, rigidez arterial ou disfunção endotelial mostraram estar consistentemente aumentados nos doentes com psoríase especialmente nos mais graves, comparativamente com o grupo controlo, observando-se na maioria dos casos correlação com o grau de inflamação. ${ }^{19-21}$ (Fig. 3)

\section{COMORBILIDADES CARDIOVASCULARES Hipertensão arterial}

A presença de prevalência aumentada de HTA em doentes com psoríase comparativamente com outros doentes dermatológicos já foi demonstrada em vários estudos. ${ }^{4,22-24}$ Uma recente meta-análise de estudos observacionais demostrou que os doentes com psoríase apresentam um risco

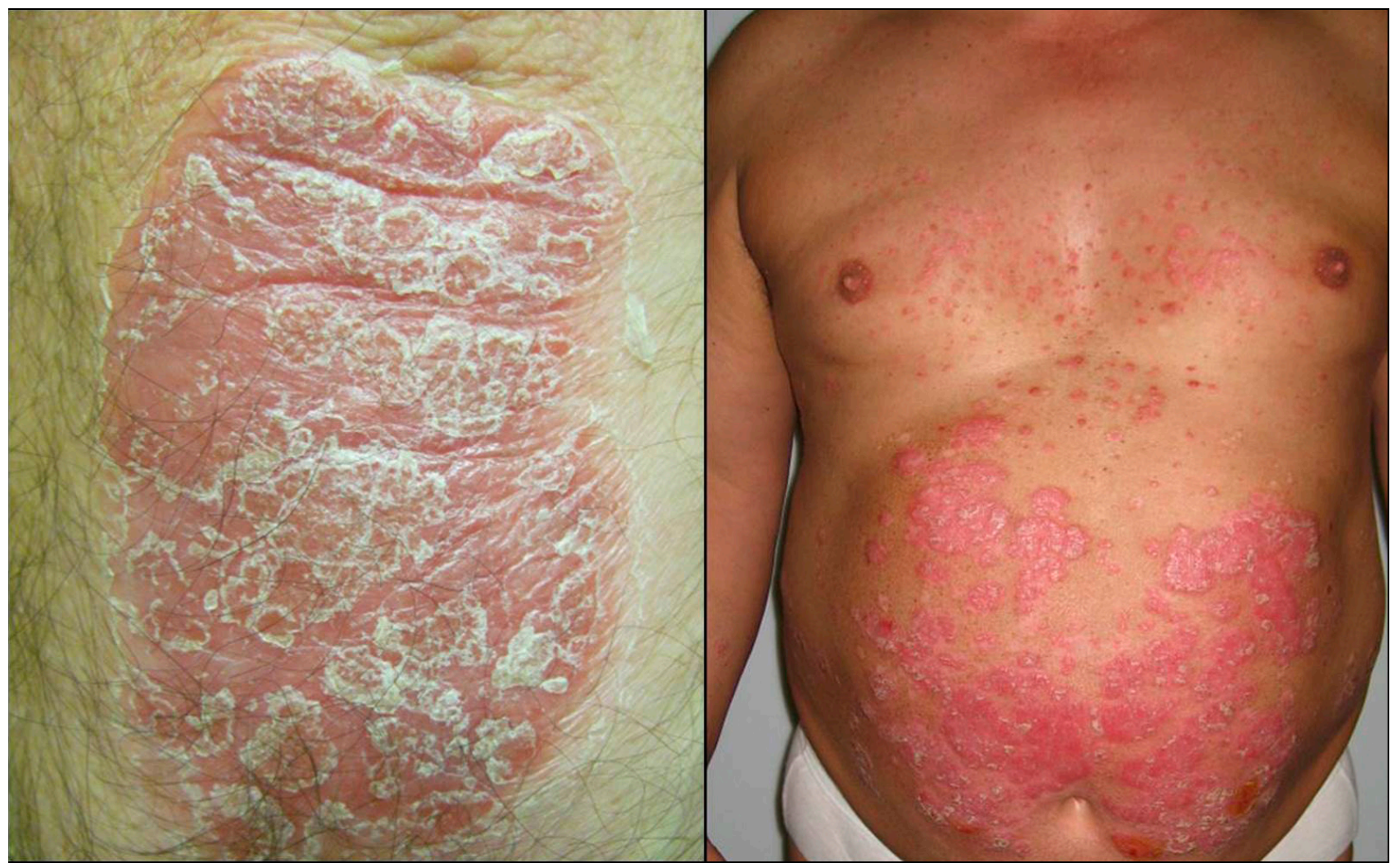

Figura 2 - Lesão característica de psoríase: placa eritematosa, bem delimitada recoberta por descamação branca-prateada; Fenótipo comum a vários doentes com psoríase grave: envolvimento superior a $10 \%$ da superfície corporal por placas de psoríase, obesidade central e presença de vários factores de risco cardiovasculares como hipertensão arterial, dislipidemia ou diabetes mellitus. 

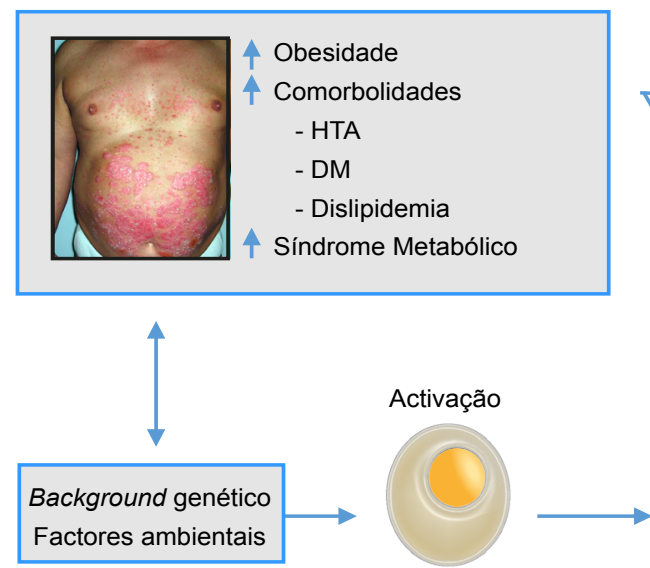

Linfócitos Th1/Th17
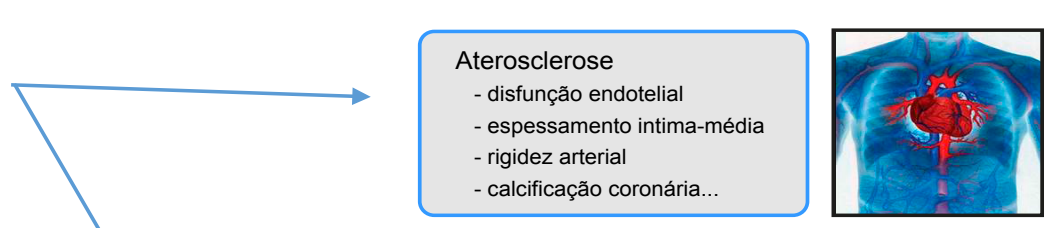
pró-inflamatórios: TNF- $\alpha$, INF-y, IL-6, $12,17,22,23$
Activação e hiperproliferação de queratinócitos e linfócitos, migração de leucócitos, angiogénese

Figura 3 - Relação entre psoríase, inflamação, comorbilidades cardiovasculares e doença cardiovascular [Adaptado de Flammer AJ, Ruschitzka F.Psoriasis and atherosclerosis: two plaques, one syndrome? Eur Heart J. 2012;33:1989-91.]

1,58 vezes superior do que a população geral de ter HTA. Adicionalmente, a psoríase grave parece conferir um risco acrescido relativamente aos casos ligeiros (OR 1,49; 95\% $\mathrm{Cl}, 1,20-1,86$ vs OR 1,30; $95 \% \mathrm{Cl} 1,15-1,47) .{ }^{24} \mathrm{~A}$ maioria dos estudos mostrou ainda uma associação independente entre HTA e psoríase após ajuste a múltiplos factores confundidores, como a idade, sexo, obesidade e tabaco. ${ }^{24}$

A razão está ainda por esclarecer, mas foram observados em doentes com psoríase, níveis aumentados da enzima conversora de angiotensina e da actividade da renina, que têm como funções regular o tónus vascular e estimular a libertação de citoquinas pró-inflamatórias, ${ }^{25,26}$ assim como de endotelina-1, um potente vasoconstritor que poderá contribuir em parte para a prevalência aumentada de HTA em doentes com psoríase. ${ }^{24}$

\section{Resistência à insulina/Diabetes mellitus}

Múltiplos estudos sugerem a possível associação entre psoríase e o aumento dos níveis séricos de glicose, hiperinsulinémia, resistência à insulina e DM tipo 2.27-29 Neimann et $\mathrm{al}^{4}$ observou uma prevalência de DM de $7,1 \%$ em doentes com psoríase grave comparativamente com $3,3 \%$ no grupo controlo. Quereshi et $\mathrm{a}^{30}$ mostrou que a psoríase estava independentemente associada com DM $(R R=1,63)$, enquanto o OR de desenvolver DM era de 2,56 e que o risco se correlacionava com a gravidade da psoríase. Além disso, vários trabalhos mostraram que a prevalência aumentada de DM nos doentes com psoríase seria independente de factores de risco tradicionais de DM, como a dislipidemia e obesidade, ${ }^{31-33}$ apesar de se saber que a obesidade e especialmente a obesidade abdominal está fortemente associada ao desenvolvimento de síndrome metabólico e DM tipo 2.

Os mecanismos potenciais para esta relação permanecem ainda um pouco por esclarecer. Boehncke et $\mathrm{a}^{34}$ mostrou uma associação significativa entre a gravidade da psoríase, a secreção de insulina e níveis séricos de resis- tina, citoquina aumentada em estados de resistência à insulina. Por outro lado é conhecido o papel do TNF-a, uma das principais citoquinas pró-inflamatórias na psoríase, na regulação da função da insulina. A indução de resistência à insulina pelo TNF- $\alpha$ poderá acontecer, pelo menos em parte, pelo seu efeito a nível dos adipócitos e hepatócitos. ${ }^{35}$ Assim, a resistência à insulina observada nos doentes com psoríase grave poderia, em parte, ser devida à inflamação crónica sistémica observada nestes doentes, conceito cada vez mais aceite. ${ }^{4}$ Adicionalmente, estudos genéticos revelaram uma associação entre loci de susceptibilidade de DM e de psoríase. ${ }^{36}$

\section{Dislipidemia}

Vários estudos observacionais e caso-controlo têm demonstrado uma associação entre psoríase e dislipidemia aterogénica, mesmo quando ajustados à idade, sexo e obesidade.,23,37-39 Os doentes psoriáticos têm concentrações plasmáticas mais elevadas de triglicerídeos, colesterol total, VLDL, LDL e lipoproteína A e igualmente concentrações séricas baixas de HDL e apolipoproteína B. ${ }^{37,40-42}$

Interessante é também a demonstração que este perfil dislipidémico se encontra presente desde o início da doença cutânea (< 1 ano) sugerindo que a dislipoproteinémia possa ser determinada geneticamente em vez de adquirida. Adicionalmente, foi demonstrado estar presente em doentes com psoríase em placa e gutata um polimorfismo genético da apolipoproteína $E$, fortemente associada com estados dislipidémicos. ${ }^{43}$ Contudo, apesar da existência de estudos genéticos que associem estas patologias, a relação fisiopatológica entre elas não foi ainda totalmente esclarecida, mas provavelmente está relacionada com a produção aumentada de citoquinas pro-inflamatórias, principalmente o TNF- $\alpha$, a leptina e a IL-6, observada nos doentes com psoríase, que têm um papel importante na regulação dos níveis de lípidos, ácidos gordos livres e colesterol. ${ }^{44-46}$ 


\section{Obesidade}

A obesidade é uma comorbilidade frequente na psoríase e vários estudos têm demonstrado que estes doentes têm mais frequentemente excesso de peso (IMC $\geq 25 \mathrm{~kg} / \mathrm{m}^{2}$ e $<30 \mathrm{~kg} / \mathrm{m}^{2}$ ) e obesidade (IMC $\geq 30 \mathrm{~kg} / \mathrm{m}^{2}$ ) quando compa-

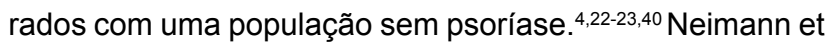
$\mathrm{al}^{4}$ observou que a prevalência da obesidade em doentes com psoríase grave era de $20,7 \%$ quando comparada com $13,2 \%$ do grupo controlo. Num ensaio clinico com etanercept com 3700 doentes a prevalência de obesidade foi de $46 \%$. Além disso, o IMC médio em 10000 doentes com psoríase moderada a grave que participaram em ensaios clinico foi de $30,6 \mathrm{~kg} / \mathrm{m}^{2}{ }^{47}$

Num estudo populacional recente em doentes com psoríase ligeira e grave, observou-se que o risco de obesidade nos doentes psoriáticos era significativamente maior comparativamente com o grupo controlo e que se associava com a gravidade da doença (OR 1,27; 95\% Cl, 1,24-1,31; e OR 1,$79 ; 95 \% \mathrm{Cl}, 1,55-2,05$ para doença ligeira e grave respectivamente). ${ }^{4}$

Apesar de haver uma forte associação entre obesidade e psoríase, é ainda controverso se a obesidade é causa ou consequência de psoríase e a etiologia desta ligação permanece ainda incerta. Sabe-se que nos doentes obesos existe um aumento dos mediadores inflamatórios, semeIhantes aos encontrados na resposta inflamatória mediada por células Th1.48 Assim, acredita-se que as citoquinas produzidas na pele possam actuar directamente sobre o tecido adiposo e vice-versa. Esta hipótese é corroborada por níveis aumentados de proteína $C$ reactiva, TNF- $\alpha$, IL-2 e IL-6, encontrados nas duas patologias. ${ }^{48,49} \mathrm{O}$ TNF- $\alpha$ leva a hiperinsulinémia através da resistência à insulina, fazendo com que as células endoteliais produzam moléculas de adesão aos monócitos, assim como reduz a síntese de adiponectina, prejudicando a sinalização da insulina. Estes elementos comuns estão envolvidos nas fases iniciais da inflamação. Deste modo, a obesidade pode potenciar a inflamação da psoríase, e simultaneamente, facilitar o desenvolvimento de síndrome metabólico. ${ }^{49}$

Existe igualmente evidência que a perda de peso tem efeito na eficácia das terapêuticas usadas no tratamento da psoríase, assim como na gravidade da doença, provavelmente por diminuir a inflamação sistémica. ${ }^{50}$

\section{Síndrome Metabólico}

A síndrome metabólica refere-se a um conjunto de factores de risco de doença cardiovasculares, que quando presentes no mesmo doente aumentam o risco cárdio-metabólico. Os indivíduos com esta síndrome têm um aumento de 3 a 9 vezes do risco de desenvolver diabetes tipo II, de 2-3 vezes de eventos cardiovasculares (EAM, AVC) e de 1,5 vezes de mortalidade cardiovascular. ${ }^{51}$

Entre os vários critérios de diagnóstico de síndrome metabólico, o National Cholesterol Education Program Adult Treatment Panel III (NCEP ATP III) é o mais utilizado nos EUA e na Europa, e define a síndrome metabólico como a presença de três ou mais dos seguintes componentes: obesidade abdominal (circunferência da cintura $\geq 102 \mathrm{~cm}$ em homens, $\geq 88 \mathrm{~cm}$ em mulheres), aumento da resistência à insulina / elevada glicemia em jejum ( $\geq 100 \mathrm{mg} / \mathrm{dL}$, ou em tratamento), diminuição da lipoproteína de alta densidade ( $<40 \mathrm{mg} / \mathrm{dL}$ em homens, $<50 \mathrm{mg} / \mathrm{dL}$ em mulheres, ou em tratamento), hipertrigliceridémia ( $\geq 150 \mathrm{mg} / \mathrm{dL}$, ou em tratamento) e hipertensão arterial (pressão arterial sistólica $\geq$ $130 \mathrm{mmHg}$, ou diastólica $\geq 85 \mathrm{mmHg}$, ou em tratamento). ${ }^{52}$

Os doentes com psoríase têm um risco aumentado de síndrome metabólico e dos seus componentes individualmente. Por exemplo, comparando uma população de doentes com psoríase grave com uma população controlo internada para cirurgia de melanoma em fase inicial observou-se que o risco de desenvolver síndrome metabólico era significativamente superior nos primeiros (OR de 5,92; 95\% $\mathrm{Cl}, 2,78-12,8) \cdot{ }^{24}$

A obesidade abdominal e a resistência à insulina são consideradas factores de risco subjacentes ao desenvolvimento de síndrome metabólico. Além disso, esta síndrome está também associada a um estado pró-inflamatório e pró-trombótico, que consiste em níveis elevados de IL6 , TNF-a, leptina, inibidor de activador de plasminogénio (PAl-1), fibrinogénio e proteína $\mathrm{C}$ reactiva, e diminuição dos níveis de anti-inflamatórios, como a adiponectina. Como referido anteriormente, entre as citoquinas inflamatórias, o TNF- $\alpha$ desempenha um papel crucial quer na psoríase, quer na síndrome metabólico. ${ }^{53-56}$

A leptina parece ter um papel central nesta ligação. Independentemente da obesidade e da síndrome metabólico, observou-se nos doentes com psoríase hiperleptinemia. Além disso, observou-se nos doentes com psoríase graves níveis séricos elevados de leptina e do receptor cutâneo da leptina. ${ }^{16,57}$

\section{Tabagismo}

Vários estudos têm demonstrado que o tabagismo é mais prevalente nos doentes psoriáticos comparativamente aos restantes doentes. ${ }^{58-61}$ A razão é desconhecida mas poderá estar relacionada com o estado de ansiedade e de depressão muitas vezes associado à psoríase. A relação entre tabaco e aumento do risco de doenças cardiovasculares está bem estabelecida, contudo a ligação entre o tabaco e a psoríase necessita de fundamentação. O fumo do cigarro expõe o corpo a substâncias potencialmente tóxicas, como é o caso da nicotina, espécies reactivas ao oxigénio e óxido nítrico, que poderão estar envolvidas na patogénese da psoríase. ${ }^{62}$

\section{Efeito do tratamento da psoríase no risco cardiovas- cular \\ O tratamento da psoríase com medicação sistémica} poderá influenciar o risco cardiovascular dos doentes. $\mathrm{O}$ metotrexato mostrou reduzir o risco de eventos cardiovasculares embora esta evidência tenha sido demonstrada especialmente em estudo observacionais de doentes com artrite reumatóide. Vários estudos demonstraram que a utilização de inibidores do TNF- $\alpha$ melhora, a curto prazo, 
alguns marcadores de risco de doença cardiovascular (por exemplo disfunção endotelial) e que a longo prazo parecem reduzir efectivamente a incidência de diabetes e de eventos cardiovasculares. ${ }^{63,64}$

Por outro lado, o tratamento dos factores de risco cardiovasculares, especialmente a obesidade, parece ter efeito na gravidade da psoríase, provavelmente por diminuição da inflamação associada à obesidade. ${ }^{65}$

\section{CONCLUSÃO}

Na última década, observou-se uma mudança drástica no conceito de doença na psoríase, deixando esta de ser vista como uma doença exclusivamente cutânea mas sim como uma doença inflamatória crónica, multissistémica, com um envolvimento muito para além da pele.

Os doentes com psoríase têm um risco aumentado de desenvolver várias comorbilidades cardiometabólicas, como hipertensão arterial, dislipidemia, resistência à insulina e obesidade, que conjuntamente com a inflamação sistémica crónica que promove uma aterosclerose precoce, é responsável pelo aumento do risco de doença cardiovascular observado nestes doentes. Esta associação a factores de risco cardiovasculares e risco aumentado de doença cardiovascular observa-se em todo o espectro de gravidade da psoríase, contudo parece ser mais importante nos doentes com psoríase grave provavelmente decorrente de um estado inflamatório mais elevado.

Embora esta associação esteja ainda por esclarecer completamente, a partilha genética e certas citoquinas pró-inflamatórias e adipocitoquinas parecem contribuir para o desenvolvimento destas comorbilidades e de doença cardiovascular.

O papel dos dermatologistas é da maior importância,

\section{REFERÊNCIAS}

1. Gudjonsson JE, Elder JT. Psoriasis: epidemiology. Clin Dermatol. 2007;25:535-46.

2. Mueller W, Herrmann B. Cyclosporin A for psoriasis. N Engl J Med. 1979;301:555.

3. Nickoloff BJ, Xin H, Nestle FO, Qin JZ. The cytokine and chemokine network in psoriasis. Clin Dermatol. 2007;25:568-73.

4. Neimann AL, Shin DB, Wang X, Margolis DJ, Troxel AB, Gelfand JM. Prevalence of cardiovascular risk factors in patients with psoriasis. J Am Acad Dermatol. 2006;55:829-35.

5. Mallbris L, Akre O, Granath F, Yin L, Lindelof B, Ekbom A, et al. Increased risk for cardiovascular mortality in psoriasis inpatients but not in outpatients. Eur J Epidemiol. 2004;19:225-30.

6. Gelfand JM, Neimann AL, Shin DB, Wang X, Margolis DJ, Troxel $A B$. Risk of myocardial infarction in patients with psoriasis. JAMA. 2006;296:1735-41.

7. Brauchli YB, Jick SS, Miret M, Meier CR. Psoriasis and risk of incident myocardial infarction, stroke or transient ischaemic attack: an inception cohort study with a nested case-control analysis. $\mathrm{Br} \mathrm{J}$ Dermatol. 2009;160:1048-56.

8. Spah F. Inflammation in atherosclerosis and psoriasis: common pathogenic mechanisms and the potential for an integrated treatment approach. Br J Dermatol. 2008;159:10-7.

9. McDonald CJ, Calabresi P. Psoriasis and occlusive vascular disease. $\mathrm{Br}$ J Dermatol. 1978;99:469-75.

10. Pearce DJ, Morrison AE, Higgins KB, Crane MM, Balkrishnan R, Fleischer $A B \mathrm{Jr}$, et al. The comorbid state of psoriasis patients in a university dermatology practice. J Dermatolog Treat. 2005;16:319-23.

11. Ena P, Madeddu P, Glorioso N, Cerimele D, Rappelli A. High prevalence não só pela possibilidade de identificar precocemente as diversas comorbilidades ou doença cardiovascular, mas também pela possibilidade de ao tratar correcta e eficazmente a psoríase e inflamação sistémica subjacente, poderem prevenir as possíveis complicações.

A abordagem destes doentes deve ser multidisciplinar, com os dermatologistas, cardiologistas, endocrinologistas e médicos de medicina geral e familiar a trabalhar conjuntamente de forma a optimizar o diagnóstico, monitorização e tratamento das diversas comorbilidades, prevenindo os eventos cardiovasculares.

Os doentes deverão ser encorajados a corrigir os factores de risco cardiovasculares modificáveis, como a obesidade, tabaco e sedentarismo e adoptarem um estilo de vida saudável, uma vez que para além de melhorarem o seu perfil cardiovascular, há hoje evidencia que tal pode resultar numa melhor resposta ao tratamento.

Por fim, são necessários estudos que permitam identificar biomarcadores analíticos e genéticos que identifiquem os doentes com psoríase em maior risco de desenvolver doença cardiovascular, no sentido de implementar medidas preventivas.

\section{AGRADECIMENTOS}

Os autores gostariam de agradecer a Berta Martins da Silva pela colaboração na realização do artigo.

\section{CONFLITO DE INTERESSES}

Sem conflitos de interesse.

\section{FONTES DE FINANCIAMENTO}

Nada a declarar.

of cardiovascular diseases and enhanced activity of the renin-angiotensin system in psoriatic patients. Acta Cardiol. 1985;40:199-205.

12. Prodanovich S, Kirsner RS, Kravetz JD, Ma F, Martinez L, Federman DG. Association of psoriasis with coronary artery, cerebrovascular, and peripheral vascular diseases and mortality. Arch Dermatol. 2009;145:700-3.

13. Ahlehoff $\mathrm{O}$, Gislason $\mathrm{GH}$, Jorgensen $\mathrm{CH}$, Lindhardsen J, Charlot M, Olesen JB, et al. Psoriasis and risk of atrial fibrillation and ischaemic stroke: a Danish Nationwide Cohort Study. Eur Heart J. 2012;33:2054-64.

14. Goulding JM, Price CL, Defty CL, Hulangamuwa CS, Bader E, Ahmed I. Erectile dysfunction in patients with psoriasis: increased prevalence, an unmet need, and a chance to intervene. Br J Dermatol. 2011;164:103-9.

15. Libby $P$. Role of inflammation in atherosclerosis associated with rheumatoid arthritis. Am J Med. 2008;121:21-31.

16. Johnston A, Arnadottir S, Gudjonsson JE, Aphale A, Sigmarsdottir AA, Gunnarsson SI, et al. Obesity in psoriasis: leptin and resistin as mediators of cutaneous inflammation. Br J Dermatol. 2008;159:342-50.

17. Cheng X, Yu X, Ding YJ, Fu QQ, Xie JJ, Tang TT, et al. The Th17/Treg imbalance in patients with acute coronary syndrome. Clin Immunol. 2008;127:89-97.

18. Ludwig RJ, Herzog C, Rostock A, Ochsendorf FR, Zollner TM, Thaci D, et al. Psoriasis: a possible risk factor for development of coronary artery calcification. Br J Dermatol. 2007;156:271-6.

19. Balci DD, Balci A, Karazincir S, Ucar E, Iyigun U, Yalcin F, et al. Increased carotid artery intima-media thickness and impaired endothelial function in psoriasis. J Eur Acad Dermatol Venereol. 2009;23:1-6.

20. De Simone C, Di Giorgio A, Sisto T, Carbone A, Ghitti F, Tondi P, et al. Endothelial dysfunction in psoriasis patients: cross-sectional case- 
control study. Eur J Dermatol. 2011;21:510-4

21. Gisondi P, Fantin F, Del Giglio M, Valbusa F, Marino F, Zamboni M, et al. Chronic plaque psoriasis is associated with increased arterial stiffness. Dermatology. 2009;218:110-3.

22. Lindegard B. Diseases associated with psoriasis in a general population of 159,200 middle-aged, urban, native Swedes. Dermatologica. 1986;172:298-304.

23. Sommer DM, Jenisch S, Suchan M, Christophers E, Weichenthal M. Increased prevalence of the metabolic syndrome in patients with moderate to severe psoriasis. Arch Dermatol Res. 2006;298:321-8.

24. Armstrong AW, Harskamp CT, Armstrong EJ. The association between psoriasis and hypertension: a systematic review and meta-analysis of observational studies. J Hypertens. 2013;31:433-42.

25. Ryder KW, Epinette WW, Jay SJ, Ransburg RC, Glick MR. Serum angiotensin converting enzyme activity in patients with psoriasis. Clin Chim Acta. 1985;153:143-6.

26. Huskic J, Alendar F, Matavulj A, Ostoic L. Serum angiotensin converting enzyme in patients with psoriasis. Med Arh. 2004;58:202-5

27. Brenelli SL, Moraes AM, Monte-Alegre S, Carvalho OM, Saad MJ. Insulin resistance in psoriasis. Braz J Med Biol Res. 1995;28:297-301.

28. Karadag AS, Yavuz B, Ertugrul DT, Akin KO, Yalcin AA, Deveci OS, et al. Is psoriasis a pre-atherosclerotic disease? Increased insulin resistance and impaired endothelial function in patients with psoriasis. Int J Dermatol. 2010;49:642-6

29. Boehncke S, Thaci D, Beschmann H, Ludwig RJ, Ackermann $\mathrm{H}$, Badenhoop $\mathrm{K}$, et al. Psoriasis patients show signs of insulin resistance. $\mathrm{Br} \mathrm{J}$ Dermatol. 2007;157:1249-51.

30. Qureshi AA, Choi HK, Setty AR, Curhan GC. Psoriasis and the risk of diabetes and hypertension: a prospective study of US female nurses. Arch Dermatol. 2009;145:379-82.

31. Ford ES, Williamson DF, Liu S. Weight change and diabetes incidence: findings from a national cohort of US adults. Am J Epidemiol. 1997;146:214-22.

32. Koh-Banerjee P, Wang Y, Hu FB, Spiegelman D, Willett WC, Rimm EB. Changes in body weight and body fat distribution as risk factors for clinical diabetes in US men. Am J Epidemiol. 2004;159:1150-9.

33. Wannamethee SG, Shaper AG, Walker M. Overweight and obesity and weight change in middle aged men: impact on cardiovascular disease and diabetes. J Epidemiol Community Health. 2005;59:134-9.

34. Gisondi P, Girolomoni G. Psoriasis and atherothrombotic diseases: disease-specific and non-disease-specific risk factors. Semin Thromb Hemost. 2009;35:313-24.

35. Ruan H, Miles PD, Ladd CM, Ross K, Golub TR, Olefsky JM, et al. Profiling gene transcription in vivo reveals adipose tissue as an immediate target of tumor necrosis factor-alpha: implications for insulin resistance. Diabetes. 2002;51:3176-88.

36. Wolf N, Quaranta M, Prescott NJ, Allen M, Smith R, Burden AD. Psoriasis is associated with pleiotropic susceptibility loci identified in type II diabetes and Crohn disease. J Med Genet. 2008;45:14-6.

37. Vanizor Kural B, Orem A, Cimsit G, Yandi YE, Calapoglu M. Evaluation of the atherogenic tendency of lipids and lipoprotein content and their relationships with oxidant-antioxidant system in patients with psoriasis. Clin Chim Acta. 2003;328:71-82.

38. Pietrzak A, Lecewicz-Torun B. Activity of serum lipase and the diversity of serum lipid profile in psoriasis. Med Sci Monit. 2002;8:CR9-CR13.

39. Akhyani M, Ehsani AH, Robati RM, Robati AM. The lipid profile in psoriasis: a controlled study. J Eur Acad Dermatol Venereol. 2007;21:1330-2.

40. Wakkee M, Thio HB, Prens EP, Sijbrands EJ, Neumann HA. Unfavorable cardiovascular risk profiles in untreated and treated psoriasis patients. Atherosclerosis. 2007;190:1-9.

41. Rocha-Pereira P, Santos-Silva A, Rebelo I, Figueiredo A, Quintanilha A, Teixeira F. Dislipidemia and oxidative stress in mild and in severe psoriasis as a risk for cardiovascular disease. Clin Chim Acta. 2001;303:33-9.

42. Reynoso-von Drateln C, Martinez-Abundis E, Balcazar-Munoz BR, Bustos-Saldana R, Gonzalez-Ortiz M. Lipid profile, insulin secretion, and insulin sensitivity in psoriasis. J Am Acad Dermatol. 2003;48:882-5.

43. Campalani E, Allen MH, Fairhurst D, Young HS, Mendonca CO, Burden $A D$, et al. Apolipoprotein $E$ gene polymorphisms are associated with psoriasis but do not determine disease response to acitretin. $\mathrm{Br} \mathrm{J}$ Dermatol. 2006;154:345-52.

44. Zuliani G, Volpato S, Ble A, Bandinelli S, Corsi AM, Lauretani F, et al.
High interleukin-6 plasma levels are associated with low HDL-C levels in community-dwelling older adults: the InChianti study. Atherosclerosis 2007;192:384-90

45. Zhang HH, Halbleib M, Ahmad F, Manganiello VC, Greenberg AS. Tumor necrosis factor-alpha stimulates lipolysis in differentiated human adipocytes through activation of extracellular signal-related kinase and elevation of intracellular cAMP. Diabetes. 2002;51:2929-35.

46. van Hall G, Steensberg A, Sacchetti M, Fischer C, Keller C, Schjerling $P$, et al. Interleukin- 6 stimulates lipolysis and fat oxidation in humans. $J$ Clin Endocrinol Metab. 2003;88:3005-10.

47. Sterry W, Strober BE, Menter A, International Psoriasis C. Obesity in psoriasis: the metabolic, clinical and therapeutic implications. Report of an interdisciplinary conference and review. $\mathrm{Br} \mathrm{J}$ Dermatol. 2007; 157:649-55.

48. Rosa DJ, Machado RF, Matias FA, Cedrim SD, Noronha FL, Gaburri $D$, et al. Influence of severity of the cutaneous manifestations and age on the prevalence of several cardiovascular risk factors in patients with psoriasis. J Eur Acad Dermatol Venereol. 2012;26:348-53.

49. Kim N, Thrash B, Menter A. Comorbidities in psoriasis patients. Semin Cutan Med Surg. 2010;29:10-5

50. Gisondi P, Del Giglio M, Di Francesco V, Zamboni M, Girolomoni G. Weight loss improves the response of obese patients with moderateto-severe chronic plaque psoriasis to low-dose cyclosporine therapy: a randomized, controlled, investigator-blinded clinical trial. Am J Clin Nutr. 2008;88:1242-7.

51. Mottillo S, Filion KB, Genest J, Joseph L, Pilote L, Poirier P, et al. The metabolic syndrome and cardiovascular risk a systematic review and meta-analysis. J Am Coll Cardiol. 2010;56:1113-32.

52. National Cholesterol Education Program Expert Panel on Detection E, Treatment of High Blood Cholesterol in A. Third Report of the National Cholesterol Education Program (NCEP) Expert Panel on Detection, Evaluation, and Treatment of High Blood Cholesterol in Adults (Adult Treatment Panel III) final report. Circulation. 2002;106:3143-421.

53. Levi M, Nieuwdorp M, van der Poll T, Stroes $E$. Metabolic modulation of inflammation-induced activation of coagulation. Semin Thromb Hemost. 2008;34:26-32.

54. Correia ML, Rahmouni K. Role of leptin in the cardiovascular and endocrine complications of metabolic syndrome. Diabetes Obes Metab. 2006;8:603-10.

55. Mojiminiyi OA, Abdella NA, Al Arouj M, Ben Nakhi A. Adiponectin, insulin resistance and clinical expression of the metabolic syndrome in patients with Type 2 diabetes. Int J Obes. 2007;31:213-20.

56. Patel DA, Srinivasan SR, Xu JH, Chen W, Berenson GS. Adiponectin and its correlates of cardiovascular risk in young adults: the Bogalusa Heart Study. Metabolism. 2006;55:1551-7.

57. Chen YJ, Wu CY, Shen JL, Chu SY, Chen CK, Chang YT, et al. Psoriasis independently associated with hyperleptinemia contributing to metabolic syndrome. Arch Dermatol. 2008;144:1571-5.

58. Herron MD, Hinckley M, Hoffman MS, Papenfuss J, Hansen CB, Callis $\mathrm{KP}$, et al. Impact of obesity and smoking on psoriasis presentation and management. Arch Dermatol. 2005;141:1527-34.

59. Setty AR, Curhan G, Choi HK. Smoking and the risk of psoriasis in women: Nurses' Health Study II. Am J Med. 2007;120:953-9.

60. Naldi L. Cigarette smoking and psoriasis. Clin Dermatol. 1998;16:571-4

61. Favato G. High incidence of smoking habit in psoriatic patients. Am J Med. 2008;121:e17.

62. Orosz Z, Csiszar A, Labinskyy N, Smith K, Kaminski PM, Ferdinandy P, et al. Cigarette smoke-induced proinflammatory alterations in the endothelial phenotype: role of $\mathrm{NAD}(\mathrm{P}) \mathrm{H}$ oxidase activation. Am J Physiol Heart Circ Physiol. 2007;292:H130-9

63. Wu JJ, Poon KY, Channual JC, Shen AY. Association between tumor necrosis factor inhibitor therapy and myocardial infarction risk in patients with psoriasis. Arch Dermatol. 2012;148:1244-50.

64. Solomon DH, Massarotti E, Garg R, Liu J, Canning C, Schneeweiss $S$. Association between disease-modifying antirheumatic drugs and diabetes risk in patients with rheumatoid arthritis and psoriasis. JAMA. 2011;305:2525-31.

65. Torres T, Selores M. Does treatment of metabolic syndrome components improve psoriasis? Report of three cases. Eur J Dermatol. 2012;22:270 